\title{
Adaptation to constant-magnitude assistive forces: kinematic and neural correlates
}

\author{
Vladimir Novakovic $\cdot$ Vittorio Sanguineti
}

Received: 14 September 2010/Accepted: 25 January 2011/Published online: 9 February 2011

(c) Springer-Verlag 2011

\begin{abstract}
In many robot-assisted rehabilitation and motor skill learning applications, robots generate forces that facilitate movement performance. While there is some evidence that assistance is beneficial, the underlying mechanisms of action are largely unknown, and it is unclear what force patterns are more effective. Here, we investigate how reaching movements (and their neural correlates) are altered by 'assistive' forces. Subjects performed center-out reaching movements, under the influence of a robot-generated force, constant in magnitude and always directed toward the target. The experimental protocol included three phases: (1) baseline (no forces), (2) force field (with two different force levels, $3 \mathrm{~N}$ and $6 \mathrm{~N}$, applied in random order), and (3) after-effect (no forces). EEG activity was recorded from motor and frontal cortical areas. In both movement kinematics and EEG activity, we looked at the effects of forces, of adaptation to such forces and at the aftereffects of such adaptation. Assistive forces initially induced a degraded performance and in general alterations in movement kinematics. However, subjects quickly adapted to the perturbation by improving their performance. With regard to EEG activity, we found (1) an increased beta band synchronization just before movements and an alpha band synchronization in the ipsilateral hemisphere, both proportional to force magnitude; (2) a gradual decrease in alpha band synchronization with
\end{abstract}

\footnotetext{
V. Novakovic $(\bowtie) \cdot$ V. Sanguineti

Department of Informatics, Systems and Telematics, University of Genoa, Via Opera Pia 13, 16145 Genoa, Italy e-mail: vladimir.novakovic@unige.it

V. Novakovic $\cdot$ V. Sanguineti

Department of Robotics, Brain and Cognitive Sciences, Italian Institute of Technology, Via Morego 30,

16163 Genoa, Italy
}

practice in the contralateral hemisphere; (3) an increase in theta band synchronization in the later stage of the force epochs; and (4) an ipsilateral to contralateral shift (from baseline to aftereffect) of theta band synchronization. These results point to the need for a careful design of assistive forces to effectively facilitate motor performance and motor learning. Moreover, EEG signals exhibit distinct features related to force and adaptation. Therefore, at least in principle, the latter might be used to monitor the learning process and/or to regulate the amount of assistance.

Keywords Reaching movements - Sensorimotor adaptation · Electroencephalography · Upper limb . Manipulandum

\section{Introduction}

Many robot-assisted rehabilitation and motor skill learning applications rely on the notion that learning can be accelerated if robots provide suitable 'assistive' forces, helping subjects to complete the task or to improve task performance-the 'guidance' hypothesis. While there is some evidence that assistive forces may be beneficial to motor learning or re-learning, at least in some situations (Reinkensmeyer and Patton 2009), the underlying mechanisms of action are still largely unknown. Moreover, even in simple tasks like reaching movements, it is unclear what form of assistance would be more effective in facilitating learning.

When subjects are exposed to a dynamic environment ('force field') that systematically perturbs arm motion, they gradually recover their original movements (Shadmehr and Mussa-Ivaldi 1994). It has been suggested that such recovery is caused by a gradual acquisition of the ability to 'predict' the disturbance, which is thus incorporated into 
the motor plan. Adaptation studies have provided a large body of knowledge on the mechanisms underlying the way the brain reacts to novel dynamic environments (Shadmehr and Wise 2005) and may provide insights into the way the nervous system represents information about the body and the environment (Thoroughman and Shadmehr 2000).

Assistive forces can be seen as peculiar types of dynamic environments, which induce a 'slacking' form of adaptation (Emken et al. 2007): if forces are helpful in accomplishing the task, they are quickly incorporated into the motor plan, and the 'voluntary' motor commands are reduced accordingly. Slacking may have adverse effects on the ability to perform the same task without assistance. To prevent this, it has been suggested that assistance should be kept to a minimum and continuously adjusted as performance improves ('assist-as-needed'). Assistance may be adjusted in terms of the observed performance (Krebs et al. 2003; Vergaro et al. 2010) or EMG activity (Krebs et al. 2003) or to reduce the human-robot interaction forces (Riener et al. 2005); see Marchal-Crespo and Reinkensmeyer (2009) for a review. At least in principle, the neural correlates of movement could be used as a source of information about the ongoing adaptation process (Daly and Wolpaw 2008). However, this requires a precise characterization of the neural correlates of different aspects of assisted performance. In particular, the ways EEG activity before or during movement reflects task difficulty, the amount of assistance, and the progress of the learning process have to be identified.

EEG studies in humans have revealed movement- and force-related brain activity in the sensorimotor and frontal areas during movement preparation, movement execution, and motor imagery. Event-related desynchronization (ERD) in the alpha band $(8-12 \mathrm{~Hz})$ has been reported in the sensorimotor areas during movement preparation, imagery and execution, in tasks like finger movements (Pfurtscheller et al. 1997) and cube manipulation (Neuper et al. 2006). Neuper and Pfurtscheller (2001) review this effect in a variety of situations: motor imagery of left- and right-hand movements, self-paced hand/finger movements, and triggered hand movements. Furthermore, when movements are triggered by an acoustic stimulus, alpha ERD was only present just after the sound (Alegre et al. 2003). In summary, many different upper limb movements induce a decrease in alpha band power, starting before the movement and finishing during or after the movement.

Event-related synchronization has also been observed in the beta band $(18-25 \mathrm{~Hz})$ just after the movement. Beta ERS is likely due to 'idling' of the motor cortex after the movement (Pfurtscheller et al. 1996) and was found to depend on the muscle mass activated (Pfurtscheller et al. 1998). In a finger movement task (Pfurtscheller and Lopes da Silva 1999) observed a high correlation between alpha and beta band ERS/ERD, and a beta ERD just before the movement.

With regard to adaptation, changes in movement-related potentials (MRPs) were reported (Makienko et al. 2005) in a finger pulling task under different types of loads. A peak in the Bereitschaftspotential portion of MRPs (600-100 ms prior to movement onset) in the prefrontal areas appears in early repetitions and disappears later. In a tool use task, Gentili et al. (2009) found that phase synchronization between different electrode sites tends to decrease with practice. EEG differences between non-skilled and skilled performers have also been reported. For instance, in a simple wrist movement task, Del Percio et al. (2010) reported that non-athletes have greater alpha ERD when compared to athletes.

Very few studies address the EEG correlates of external forces. A common observation is that cortical motor areas are synchronized with muscle activity in the beta band during a static force task (Rearick et al. 2001; Witte et al. 2007; Chakarov et al. 2009), and in the gamma band during a dynamic force task (Andrykiewicz et al. 2007). On the other hand, Slobounov et al. (2002) reported that both force and end effector movements affect movement-related potentials (MRP). Particularly, they found that only when the index finger was used, the level of force affected the MRP, whereas for the other fingers there were no MRP changes. However, the effect of force per se was not reflected in the EEG signal.

Aspects related to task difficulty, like memory, learning, workload, or attention, have been associated (Klimesch 1999) to theta band activity in the frontal cortex. More specifically, an increase in the theta band power was observed during different memory tasks (Klimesch et al. 1996; Gevins et al. 1997; Ishii et al. 1999; Jensen and Tesche 2002). Learning (Caplan et al. 2003) as well as attentional processing (Ishii et al. 1999) were also found to induce a theta power increase.

Here, we investigate how reaching movements are altered by constant-magnitude forces, directed toward the target. We look at both movement kinematics and their EEG correlates in frontal and sensorimotor areas. We specifically focus on the effects of both force magnitude and practice (adaptation) and look at the overall effect on both performance and neural activity when the assistive forces are finally removed.

\section{Materials and methods}

Task and experimental protocol

A planar manipulandum with two degrees of freedomBraccio di Ferro; see Casadio et al. (2006) for details—was 
used to generate assistive forces and to record hand positions. Subjects sat on a chair, with their torso and wrist restrained by means of suitable holders, and grasped the handle of the manipulandum with their dominant hand. A light support allowed the forearm to slide on the horizontal table surface with a low friction. In this way, movements were restricted to the horizontal plane, with no influence of gravity. Seat position was also adjusted so that, with the cursor pointing at the center of the workspace, the elbow joint was anteflexed about $90^{\circ}$, and the shoulder was horizontally abducted about $45^{\circ}$, while the arm was kept approximately horizontal, at shoulder level. A $19^{\prime \prime}$ LCD computer screen was placed in front of the subjects, about $1 \mathrm{~m}$ away, at eye level. The current position of the hand was continuously displayed, as a yellow circle on a black background. The target was also displayed, as a green round circle (diameter $2 \mathrm{~cm}$ ). The visual scale factor was $1: 1$. Subjects had to perform center-out reaching movements, under visual control; starting from the same central position and moving toward four randomly selected directions $\left(45^{\circ}, 135^{\circ}, 225^{\circ}\right.$ and $\left.315^{\circ}\right)$. Target distance was $0.1 \mathrm{~m}$ from the center (reference) position.

The experiment was organized into epochs, each consisting of twelve repetitions of each of the four directions (a total of $12 \times 4=48$ movements). During each epoch, the robot either generated no force (null trials) or a force of constant magnitude, directed toward the target ('force' trials). In force trials, force started when the cursor was at least $1 \mathrm{~cm}$ away from the start position and ended at $9 \mathrm{~cm}$ from the same position. Therefore, subjects had to start the movement in order to trigger the force and the force did not interfere with movement completion. This implied an active involvement of the subjects, e.g. they could not rely just on the force to move them from the start position to the end position (target), but they had to incorporate the force in the motor plan.

The experimental protocol consisted of four phases: (1) familiarization - the robot generates no force; 1 epoch, i.e. 48 movements; used to familiarize the subject with the apparatus and not included in the subsequent data analysis; (2) baseline (BAS)—no force, 1 epoch, i.e. 48 movements; (3) force-the robot generates a constant force, 2 epochs, i.e. 96 movements; and (4) after-effect (AE) - no force, 1 epoch, i.e. 48 movements. Therefore, the whole experiment consisted of five epochs, i.e. 240 movements. During force trials, we used two different force magnitudes: 'low' $(3 \mathrm{~N})$ and 'high' $(6 \mathrm{~N})$ so that each subject was exposed first to one level of force and then to the other. Force was kept constant within one epoch, and the low-high sequence was randomized across subjects (some subjects had low force first and high force later; and vice versa) in order to cancel order effects.

In addition to movement kinematics and its changes, we also looked at changes in EEG activity. Since many of the previous studies suggest that motor planning and execution mostly involve the frontal and sensorimotor areas of brain cortex, eight gold electrodes, arranged on an electrode cap (g.EEGcap, g.tec, Guger Technologies OEG, Graz, Austria), were placed in the following sites (International 10-20 electrode system) on the subject's scalp: central (C3, $\mathrm{C} 1, \mathrm{Cz}, \mathrm{C} 2, \mathrm{C} 4)$ and frontal $(\mathrm{F} 3, \mathrm{Fz}, \mathrm{F} 4)$ lobe. The left earlobe was used as a reference. The handle of the robot was electrically isolated to reduce noise in EEG recordings. EEG signals were band-pass filtered $(0.1-100 \mathrm{~Hz})$ and amplified by means of a multi-channel EEG amplifier (g.BSamp, g.tec, Guger Technologies OEG, Graz, Austria).

To allow monitoring, both motor preparation and movement start, target onset and movement start were separated by a 3-s interval. Each trial started when subjects moved the yellow hand cursor to the center of the screen (green circle); see Fig. 1 (top left). At this point, a 'cue' signal appeared (an arrow showing the direction of the next target)—see Fig. 1 (top middle)—which lasted for $3 \mathrm{~s}$ ("preparation"). Then, the arrow was turned off, the target appeared (GO signal) and subjects could initiate the movement ("movement"); see Fig. 1 (top right). During the "preparation" interval, subjects were instructed to look at the arrow with their peripheral gaze and when the cue was not on, a small rectangle was shown to reduce eye movements. When the target appeared, subjects were instructed to perform fast and accurate movements toward it.

The experiment involved a total of ten subjects $(9 \mathrm{M}+1 \mathrm{~F})$ with no signs of neurological problems. The average age was $24 \pm 4$ years. All subjects were righthanded. Subjects were randomly assigned to two groups (low-high and high-low) that only differed in the order of presentation of force magnitudes. The research conforms to the ethical standards laid down in the 1964 Declaration of Helsinki that protects research subjects. Each subject signed a consent form that conforms to these guidelines.

\section{Data analysis}

Movement trajectories were sampled at $100 \mathrm{~Hz}$ and stored for subsequent analysis. Kinematic data were smoothed using a 4th order Savitzky-Golay filter, with a 270-ms time window (equivalent cut-off frequency: $7.5 \mathrm{~Hz}$ ). We used the same filter to estimate the velocity as the first derivative of the trajectory. We identified the time instants corresponding to movement start and the first stop as the instants when the speed went, respectively, above and below a $0.02 \mathrm{~m} / \mathrm{s}$ threshold. For each trajectory, we computed the linearity index (percent increase in the actual trajectory length with respect to the straight line from the center to the target), the endpoint error (distance between the target center and the hand position at stop), the peak speed 

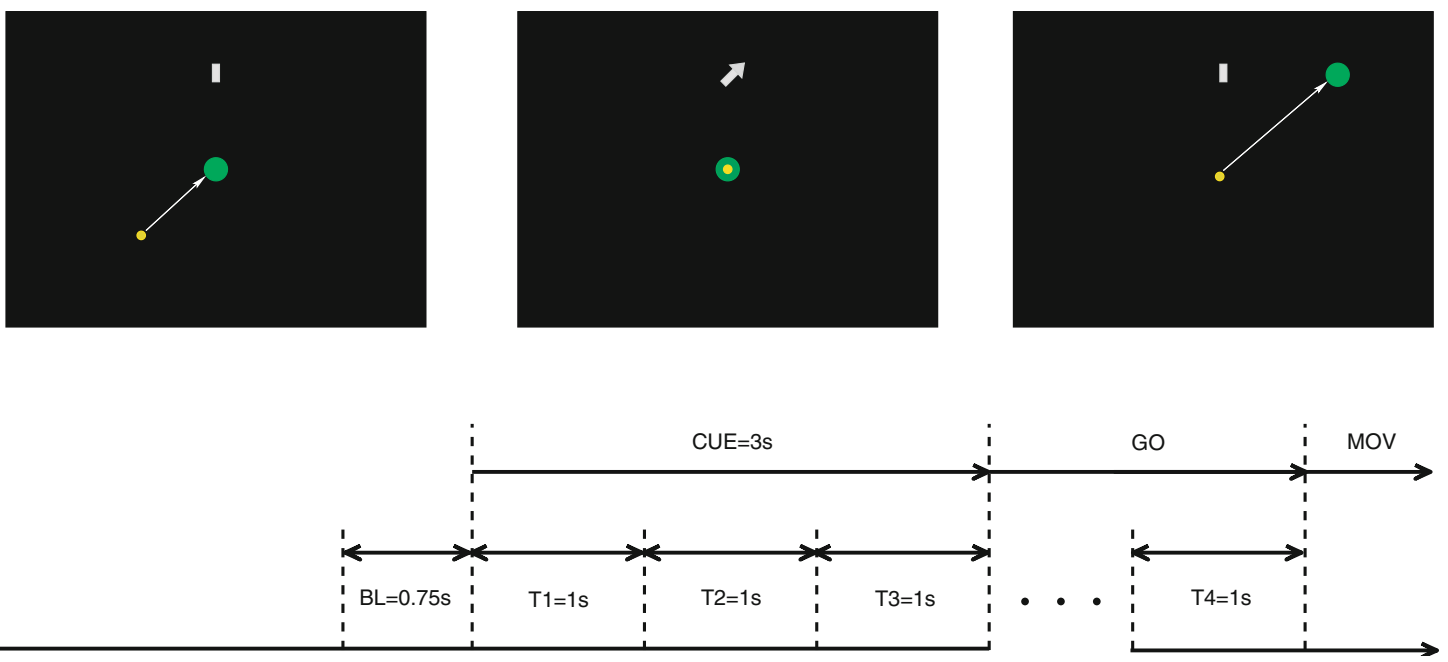

Fig. 1 Structure and timeline of one individual trial. To start a trial, subjects had to move the yellow cursor to the center of the screen (top left). Then, a CUE signal appeared (an arrow showing the direction of the next target) (top middle). The cue lasted for $3 \mathrm{~s}$ (preparation), then the target appeared (GO signal, top right) and subjects were instructed

(maximum of the velocity norm during the movement), and the movement duration (time interval between movement start and stop). Similarly, we defined the durations of the acceleration and the deceleration portions of the movement. Analysis of the kinematic data was conducted using MATLAB (Mathworks Inc.).

The EEG signals were sampled at $256 \mathrm{~Hz}$ (National Instruments NIDAQ 6040-E) through a separate computer and stored for later analysis. Synchronization of the two acquisition systems was achieved by using a direct connection between the two computers' I/O cards. The robot PC generated a TTL synchronization signal (on during CUE period, off otherwise), which was acquired by one of the analog channels of the I/O card of the computer used to acquire EEG signals.

Offline processing of the EEG data was conducted using the EEGLAB package (sccn.ucsd.edu/eeglab/) based on MATLAB. The recordings were low-pass filtered with a 20th order two-way least-squares FIR filter, with a cut-off frequency of $35 \mathrm{~Hz}$-based on the EEGLAB function 'eegfilt()' - and pre-processed by removing mean and linear trends. Corrupted data from individual movements were identified by visual inspection. These trials were completely removed from further analysis. Likewise, we eliminated from analysis all the trials that were performed incorrectly: anticipated starts (when subjects moved before the target appeared), late starts (when subjects did not start the movement within the given time frame of $600 \mathrm{~ms}$ ) and abnormally long durations (when subjects moved very slowly or were distracted in general causing unusually long movements). Overall, we eliminated an average $20 \pm 5 \%$ to move. For purposes of analysis, the trial was divided into four 1-s time intervals (T1-T4). A 0.75 -s interval just before the CUE was used as baseline $(B L)$ to calculate relative EEG power changes. Note that, depending on the subject's reaction time, T3 and T4 could partly overlap

of all trials. However, $10 \pm 5 \%$ were in the initial phase of the experiment (familiarization), which was not taken into consideration for analysis. Hence, only $5-10 \%$ of the analyzed trials were discarded.

Independent component analysis (ICA)_ "runica" (Bell and Sejnowski 1995) and "fastica" (Hyvarinen 1999) algorithms - was then applied to the signals within each individual epoch to remove muscular or eyeblink artifactrelated components. ICA was used iteratively, by identifying components associated to artifacts and removing them until the signal appeared to be artifact free. Individual trials were then aligned to the GO signal (Fig. 1) into 5-s intervals $(-4 \mathrm{~s}<t<1 \mathrm{~s})$.

Finally, the EEG signals were band-pass filtered to identify the contributions in the theta $(4-8 \mathrm{~Hz})$, alpha $(8-12 \mathrm{~Hz})$, and beta $(18-25 \mathrm{~Hz})$ bands. As in Pfurtscheller and Lopes da Silva (1999), we then estimated the relative event-related synchronization (ERS) or desynchronization (ERD) as the percent change of the signal power $P$, relative to that of the signal recorded during a reference period, $R$ :

$\mathrm{ERD}=100 \times \frac{(P-R)}{R}$

As reference period for each trial, we took the 0.75-s time interval immediately before the CUE: $t_{\text {cue }}-0.75 \mathrm{~s}<$ $t<t_{\text {cue }}$, where $t_{\text {cue }}=-3 \mathrm{~s}$. We then calculated ERS/ERD for three 1-s intervals starting from $-3 \mathrm{~s}$ and finishing with movement start, namely from $-3 \mathrm{~s}<t<-2 \mathrm{~s}$ (T1, afterCUE), $-2 \mathrm{~s}<t<-1 \mathrm{~s}$ (T2), $-1 \mathrm{~s}<t<0 \mathrm{~s}$ (T3, beforeGO), as well as for a 1-s interval just before movement start (T4, before-MOV); see Fig. 1. 
Statistical analysis

A four-way ANOVA was performed on the kinematic data. More specifically, we tested the influence of force magnitude $(0 \mathrm{~N}, 3 \mathrm{~N}$ and $6 \mathrm{~N})$, target direction $\left(45^{\circ}, 135^{\circ}, 225^{\circ}, 315^{\circ}\right)$ and phase (early and late) on the linearity index, endpoint error, peak speed and movement duration, for the two groups of subjects (low-high and high-low). Phase was defined as the first half (early) and the second half (late) of each epoch, i.e. first and second 24 trials within a 48-trial epoch.

With regard to EEG recordings, we performed a fourway ANOVA on the ERS/ERD values for each band (theta, alpha and beta) with EEG channels, phase (early and late) and force $(3 \mathrm{~N}$ and $6 \mathrm{~N})$ as factors for different groups (low-high and high-low). Direction dependence was not taken into consideration (the number of trials was too low to allow reliable test of the direction effect). Instead, we focused on force and adaptation effects.
In addition, we ran a three-way ANOVA to test the ERD/ERS changes between the BAS and AE epochs. Phase (BAS vs. AE), group (low-high and high-low), and channel were the factors. In this case, for each phase we took the whole epoch, i.e. 48 trials.

Post hoc statistical analysis was conducted using Tukey Honest Significant Difference (HSD) test.

\section{Results}

Kinematic analysis

For a typical subject (from the high-low group), Fig. 2 depicts the observed trajectories, the speed profiles and the time course of the endpoint error.

The results of statistical analysis are summarized in Table 1 for all parameters.

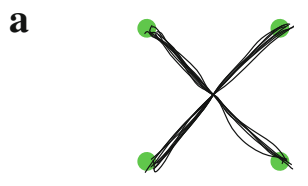

BAS

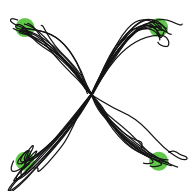

$\mathrm{F}=6 \mathrm{~N}$

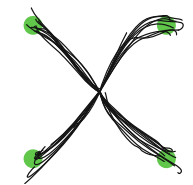

$\mathrm{F}=3 \mathrm{~N}$

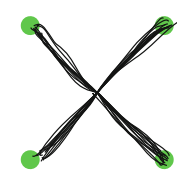

$\mathrm{AE}$
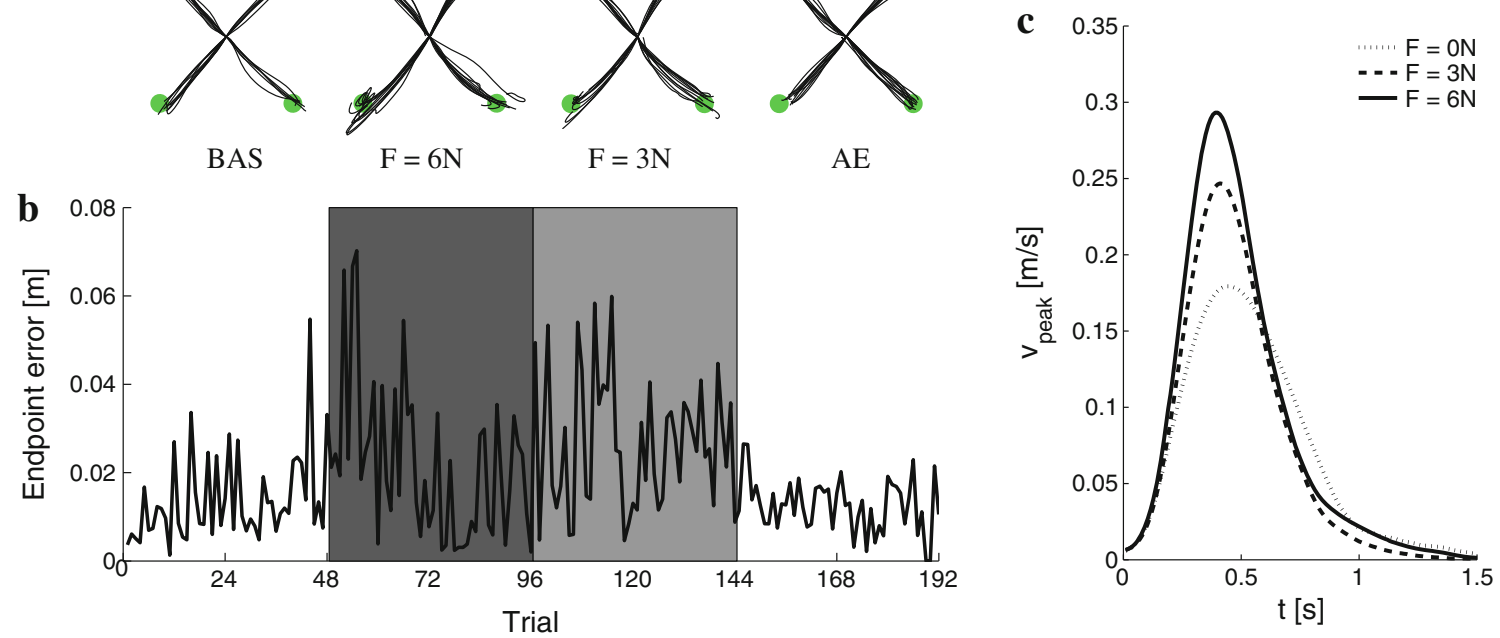

Fig. 2 Movement kinematics for a typical subject in the high-low group. a Typical hand trajectories during four different epochs: $0 \mathrm{~N}$ (BAS), $3 \mathrm{~N}, 6 \mathrm{~N}$, and $0 \mathrm{~N}$ (AE). b Trial-by-trial evolution of the endpoint error (light and dark areas correspond to the 'low'- and 'high'-force magnitudes, and white areas correspond to $0 \mathrm{~N}$ (BAS and $\mathrm{AE}$ ) epochs. c Speed profiles for $0 \mathrm{~N}$ (BAS; dotted line), $3 \mathrm{~N}$ (dashed), and $6 \mathrm{~N}$ (solid)

Table 1 Statistical analysis on kinematic indicators

\begin{tabular}{lclllll}
\hline Effect & Linearity index & Endpoint error & Peak speed & Movement duration & Deceleration duration & Acceleration duration \\
\hline Force & $0.0033^{* *}$ & $0.009^{* *}$ & $<0.00001^{* *}$ & $<0.00001^{* *}$ & $0.0002^{* *}$ & $0.0005^{* *}$ \\
Phase & 0.23 & $0.0008^{* *}$ & $0.02^{*}$ & 0.2 & 0.27 & 0.21 \\
Direction & $<0.00001^{* *}$ & 0.06 & $<0.00001^{* *}<0.00001^{* *}$ & $0.00002^{* *}$ & $<0.00001^{* *}$ \\
Force $\times$ phase & $0.0002^{* *}$ & 0.98 & 0.4 & 0.32 & 0.52 & 0.08 \\
Force $\times$ direction & 0.11 & $0.009 * *$ & $<0.00001 * *$ & $0.001 * *$ & $0.0003^{* *}$ & 0.18 \\
Phase $\times$ direction & $0.002^{* *}$ & 0.58 & $0.0001^{* *}$ & 0.51 & 0.54 & 0.79 \\
Force $\times$ phase $\times$ direction & $0.008^{* *}$ & 0.23 & $0.02^{* *}$ & 0.51 & 0.44 & 0.052
\end{tabular}

Significant $(P<0.05)$ and highly significant $(P<0.01)$ effects are denoted with $*$ and $* *$, respectively. The effect of group (and the related interactions) is never significant and has been omitted for simplicity 
The group effect and all group-related interactions are not reported as they were never significant. In other words, the effects of force and adaptation do not depend on the order of presentation of the force levels.

\section{Path curvature}

The linearity index is commonly assessed in force adaptation tasks, because alterations in path curvature are expected when force is perpendicular to movement direction. However, Fig. 2a suggests that even in this experiment, in the force trials the trajectories tend to be more curved.

In fact, statistical analysis revealed a significant effect of force $(F(2,16)=4.16, P<0.0033)$ and a significant force $\times$ phase interaction $(F(2,16)=8.91, P<0.0002)$. On the other hand, the effect of phase was non-significant, which indicates that curvature did not systematically change with repeated practice. Post hoc analysis revealed that all pairwise differences for different forces were significant: $0 \mathrm{~N}$ versus $3 \mathrm{~N}(P<0.05), 0 \mathrm{~N}$ versus $6 \mathrm{~N}$ $(P<0.001)$, and $3 \mathrm{~N}$ versus $6 \mathrm{~N}(P<0.03)$.

Moreover, the same force effect is significant in both early and late phases, taken separately, with an exception for the early $0 \mathrm{~N}$ case that did not differ significantly from the $3 \mathrm{~N}$ case. However, in the case of $0 \mathrm{~N}$ force, we found that path curvature in the early phase is significantly greater than that observed in the late phase $(P<0.005)$. Overall, these results suggest that curvature increases with force magnitude; see also Fig. 3a).

Furthermore, the directional patterns of curvature observed in the force trials are a magnification of those observed in baseline trials. This observation is confirmed by the examination of the directional patterns of peak acceleration (i.e. the maximum acceleration observed in the first half of the movement). Figure $3 b$ shows that all movements exhibit the same directional patterns of peak acceleration and assistive forces only have a scaling effect. In other words, assistive forces tend to amplify the natural trend of baseline movements to accelerate the arm away from target direction.

In addition, we found statistically significant effects of direction, phase $\times$ direction and force $\times$ phase $\times$ direction (see Table 1). These effects are likely due to differences in arm inertia and/or geometry and will not be discussed in detail for this and all subsequent parameters. The interested reader is referred to Table 1 for a complete account of these results.

\section{Endpoint error}

Endpoint error can be taken as a measure of the subjects' reaching performance. Figure 4 a suggests that this quantity changes with both force magnitude and practice.

Statistical analysis confirmed these observations: a significant effect of force $(F(2,16)=6.14, P<0.01)$ and a significant effect of phase $(F(1,8)=24.2, P<0.001)$ : endpoint error increases with force magnitude and decreases with practice.

Post hoc analysis only showed significant differences between $0 \mathrm{~N}$ versus $6 \mathrm{~N}$ comparison $(P<0.009)$. On the other hand, the $3 \mathrm{~N}$ versus $6 \mathrm{~N}$ comparison was just above the significance level $(P>0.05)$, whereas $0 \mathrm{~N}$ versus $3 \mathrm{~N}$ was non-significant $(P>0.55)$. Moreover, phase was significant for all the force levels: $0 \mathrm{~N}(P<0.02), 3 \mathrm{~N}$ $(P<0.05)$, and $6 \mathrm{~N}(P<0.05)$.

\section{Peak speed}

Figure $4 \mathrm{~b}$ displays the effects of force and adaptation on peak speed. While there is a clear effect of force, the effect of adaptation is only visible in 'low' forces.
Fig. 3 Effect of force magnitude. a Path curvature, quantified by the linearity index (mean $\pm \mathrm{SE}$ for all subjects) for the three phases: $0 \mathrm{~N}$ (BAS), $3 \mathrm{~N}$, and 6 N. Solid and dashed lines correspond to early and late phase, respectively. b Peak acceleration for one typical subject, for all four directions and all levels of force: $0 \mathrm{~N}$ (light-gray), $3 \mathrm{~N}$ (dark-gray), and $6 \mathrm{~N}$ (black). Dashed lines indicate the directions corresponding to straight line movements toward each of the four targets. Scale $6 \mathrm{~m} / \mathrm{s}^{2}$
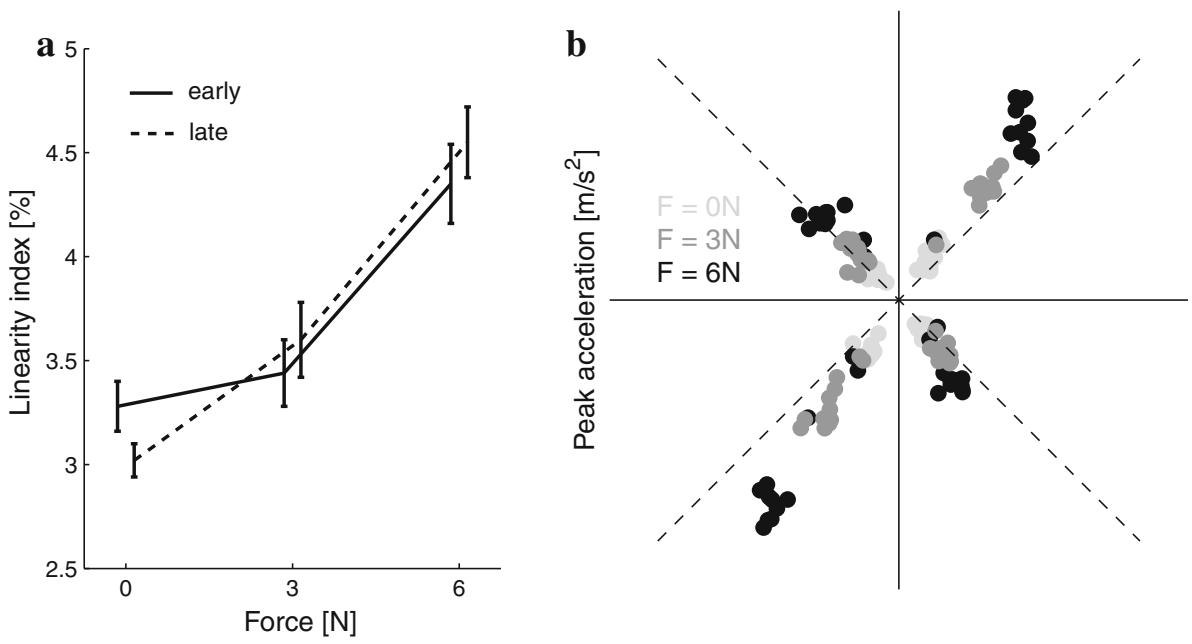
Statistical analysis confirmed a significant effect of force $(F(2,16)=39.09, P<0.000001)$. The effect of phase was significant as well $(F(1,8)=8.53, P<0.02)$.

Post hoc analysis showed that all the pairwise differences for different forces were significant: $0 \mathrm{~N}$ versus $3 \mathrm{~N}$ $(P<0.0003), 0 \mathrm{~N}$ versus $6 \mathrm{~N}(P<0.0001)$, and $3 \mathrm{~N}$ versus $6 \mathrm{~N}(P<0.006)$. On the other hand, the effect of phase was non-significant when the different force magnitudes were taken separately.

\section{Movement duration}

Movement duration also exhibited a strong dependence on force, confirmed by statistical analysis $(F(2,16)=22.76$, $P<0.000001)$. Post hoc analysis clarified that movement duration is significantly different in $0 \mathrm{~N}$ versus $3 \mathrm{~N}$ $(P<0.00015)$ and $0 \mathrm{~N}$ versus $6 \mathrm{~N}(P<0.00014)$, but not in $3 \mathrm{~N}$ versus $6 \mathrm{~N}$ conditions.

In contrast, we observed no consistent effect of adaptation, even though Fig. 4c suggests that some effect of adaptation, at least in 'high'-force epochs.

Further data analysis confirmed that the changes observed in total duration occur in both the acceleration and deceleration portions of the movements.

Overall, the above results suggest that movement kinematics is heavily affected by constant forces (greater endpoint error, curvature and peak speed; decreased movement duration). However, movements quickly adapt to these perturbations (endpoint error decreases).

\section{EEG analysis}

We focused on changes in event-related synchronization (ERS) or desynchronization (ERD) in the theta, alpha, and beta bands, for different force levels and for different phases. In particular, we compared force epochs in terms of force magnitude (high, low) and phase (early, late). The analysis was performed for four different time intervals: after-CUE (T1), T2, before-GO (T3), and before-MOV
(T4). In addition, we compared the baseline versus aftereffect epochs (again, in intervals T1-T4) to look for effects of 'assisted' practice.

\section{Theta band (4-8 Hz)}

Theta band activity exhibited no significant force effects. The only significant effect was a force $\times$ group interaction $(F(1,8)=17.57, P<0.002)$ in the after-CUE (T1) period. Post hoc analysis clarified that theta synchronization increased (from ERD to ERS) in the second force epoch, in both low-high $(P<0.05)$ and high-low $(P<0.03)$ groups, see Fig. 5. In other words, theta activity increases with practice, irrespective of the magnitude of the force.

A similar trend was also found in T2 (significant force $\times$ group interaction; $P<0.05$ ). However, post hoc analysis showed that such increase was not significant in neither of the groups when taken separately. No significant effects were found in the remaining time intervals (T3, T4).

\section{Alpha band (8-12 Hz)}

With regard to the after-CUE (T1) interval, we found a significant effect of channel $(F(7,56)=2.11, P<0.05)$, as well as significant channel $\times$ phase $(F(7,56)=2.22$, $P<0.05)$ and channel $\times$ force $\times$ phase $(F(7,56)=2.27$, $P<0.04)$ interactions.

Post hoc comparisons revealed a significant phase effect (from ERS - early - to ERD-late) in contralateral (C1) motor areas $(P<0.05)$. Moreover, we observed a significant force effect (greater synchronization for 'low' forces) in ipsilateral $(\mathrm{C} 2)$ motor areas $(P<0.04)$. Other channels showed no statistically significant changes.

We additionally found a significant $(P<0.005)$ channel effect in the before-MOV (T4) interval. However, post hoc analysis revealed no significant differences among individual recording sites. In all other time intervals (T2, T3), no significant effects were observed.
Fig. 4 Effect of adaptation (mean \pm SE for all subjects). a Endpoint error, b peak speed and $\mathbf{c}$ movement duration. Solid and dashed lines correspond, respectively, to the early and late portion of each phase $(0 \mathrm{~N}$ (BAS), $3 \mathrm{~N}$ (low), and $6 \mathrm{~N}$ (high) force)
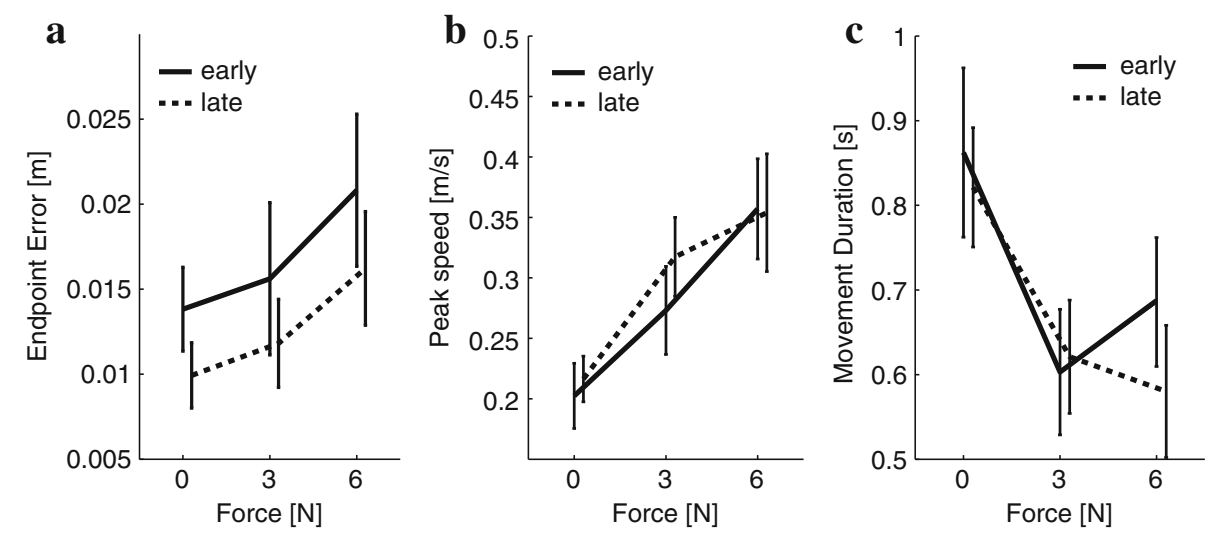

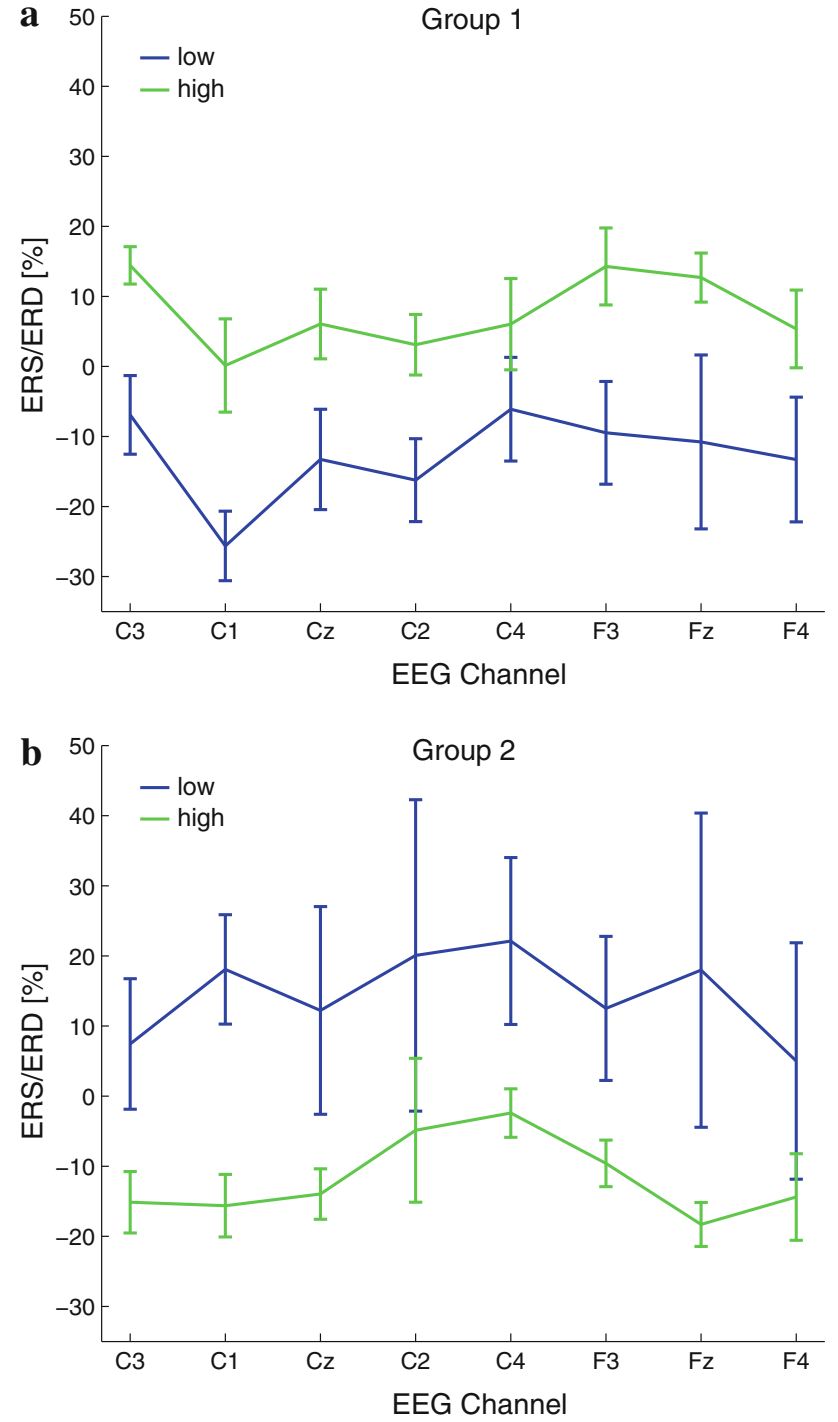

Fig. 5 Theta band synchronization in the after-CUE (T1) interval for each recording site (mean $\pm \mathrm{SE}$ for all subjects), during low (blue) and high (green) force epochs, for groups low-high (a) and high-low (b). Theta band power increases in the later force epoch with disregard to the force order

\section{Beta band $(18-25 \mathrm{~Hz})$}

Figure 6 shows the power changes in the beta band in the before-MOV (T4) time interval. These results suggest a significant effect of force (ERD for low force, ERS for high force).

Statistical analysis confirmed that the effect of force was, in fact, significant $(F(1,8)=51.08, P<0.0001)$. In contrast, we found no significant phase effects. Furthermore, significant effects of force were observed in the other time intervals (T2, T3). These effects follow the same trend as in T4, but the $P$-values were higher $(P<0.05$ and $P<0.01$, respectively). In $\mathrm{T} 1$, these same effects were non-significant.

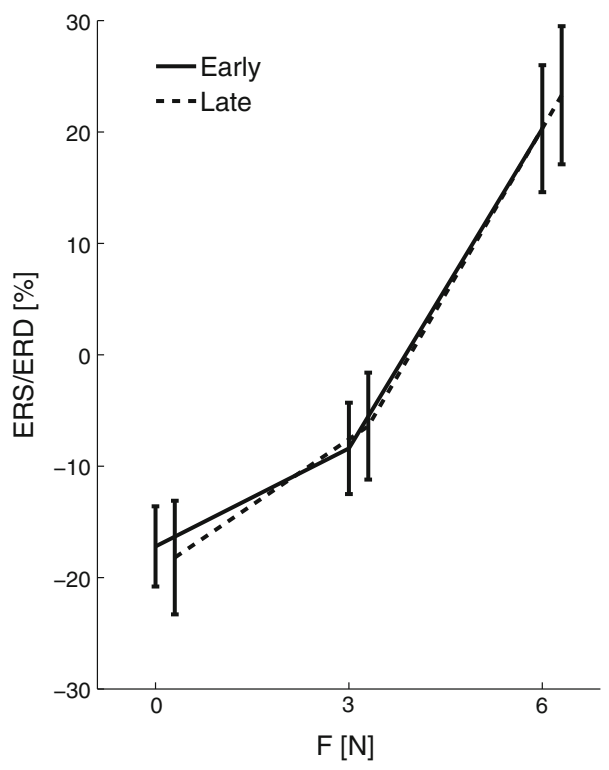

Fig. 6 Beta band synchronization in the before-MOV (T4) interval (mean \pm SE for all subjects and recording sites). Solid and dashed lines correspond, respectively, to the early and late portion of each phase

Post hoc analysis confirmed the significance of the force effect: $0 \mathrm{~N}$ versus $3 \mathrm{~N}(P<0.05), 0 \mathrm{~N}$ versus $6 \mathrm{~N}$ $(P<0.00012)$ and $3 \mathrm{~N}$ versus $6 \mathrm{~N}(P<0.0002)$ in the T4 interval. Overall, these results indicate that beta activity just before the movement is directly related to force magnitude.

In summary, EEG recordings exhibited a gradual increase in theta activity during the 'force' epochs, irrespective of phase or force magnitude, in the after-CUE interval. In this same interval, alpha activity exhibited a decrease with practice in contralateral motor areas $(\mathrm{C} 1)$, and an increase in ipsilateral motor areas (C2) with lower forces. With regard to the before-MOV (T4) time interval, we found that beta activity was significantly greater at 'high'-force levels.

In relation to inter-subject variability, each of the above effects was observed in at least 8 individual subjects (out of $10)$.

In order to assess whether and how EEG activity was modified by 'assisted' practice, we analyzed the differences between baseline and after-effect phases.

\section{Theta band (4-8 Hz)}

In the after-CUE (T1) interval, significant changes were observed between the activities of the left (contralateral) and right (ipsilateral) hemisphere. ANOVA confirmed that interaction channel $\times$ phase was significant $(F(7,56)=$ 2.91, $P<0.01)$. Post hoc analysis revealed that in the after-effect phase, synchronization increased in the 
contralateral hemisphere $(\mathrm{C} 1, \mathrm{C} 3$, and $\mathrm{F} 3)$ and decreased in the ipsilateral hemisphere ( $\mathrm{C} 4$ and $\mathrm{F} 4)$. No significant effects were observed in the other time intervals (T2-T4).

\section{Alpha band $(8-12 \mathrm{~Hz})$}

In the alpha band, we found a significant decrease in ERS/ ERD in both T3 $(F(1,8)=6.92, P<0.03)$ and T4 $(F(1,8)=5.59, P<0.05)$ intervals, but not in $\mathrm{T} 1$ and $\mathrm{T} 2$. Post hoc analysis showed that the effect of phase was present overall, while looking at the activity at the individual sites no significant differences were observed.

\section{Beta band (18-25 Hz)}

Beta band activity after-CUE (T1) exhibited a greater activity in the after-effect period as compared to the baseline. Statistical analysis confirmed the significance of this effect $(F(1,8)=6.39, P<0.035)$. As in alpha band, we found no significant effect of phase when each channel was separately analyzed. The other time intervals showed no significant changes.

In summary, the comparison of baseline and after-effect phases revealed a significant increase in activity in the beta band after-CUE and a significant decrease in activity in the alpha band before-GO and before-MOV (both effects present in all the subjects).

Moreover, in the theta band the contralateral and the ipsilateral hemispheres seem to 'switch' their roles (from ERD to ERS in contralateral hemisphere, from ERS to ERD in ipsilateral). Only one subject exhibited an overall nonlocalized increase in synchronization (from ERD to ERS).

\section{Discussion}

The aim of this study was to investigate how movement kinematics and movement-related EEG activity during reaching movements are altered by changes in the dynamic environment. The dynamic environment consisted of a force of constant magnitude, directed toward the movement target: an 'assistive' environment commonly used in robotassisted rehabilitation protocols, see for instance (Casadio et al. 2009).

More specifically, we looked at how movement kinematics and EEG activity change with force magnitude and at kinematic and neural correlates of adaptation to such forces.

\section{Movement kinematics}

From the kinematic point of view, the main findings were that (1) constant-magnitude 'assistive' forces substantially alter movement kinematics, in a way that depends on force magnitude and (2) a prolonged exposure to assistive forces elicits adaptation, in the sense that movements tend to revert to the unperturbed situation.

\section{Constant-magnitude assistive forces alter movement kinematics}

When reaching movements are performed under the action of a constant-magnitude assistive force, trajectories become more curved, and endpoint error and peak speed are greater than in no-force conditions. Both effects increase with force magnitude.

These results can be simply explained by the fact that attractive forces result in an extra acceleration of the hand, so that the latter achieves a greater speed; see Figs. 3 and 4. Likewise, at the end of the movement the hand is more difficult to stop; see Fig. 2.

These phenomena (extra initial acceleration, greater difficulty to stop) have opposite effects on movement duration: at lower forces, the facilitating (acceleration) effect prevails and the overall movement duration decreases. In contrast, at high forces the difficulty to stop prevails, and duration tends to be greater (but still less than in the no force situation); see Fig. 4.

A less obvious effect is observed in trajectory curvature-trajectories are more curved when force is present. A look at trajectories (see Fig. 2) suggests that forces tend to amplify the directional error that was already present in unperturbed trajectories (Gordon et al. 1994b), but do not change its peculiar direction-dependent pattern. Moreover, the effect increases with force magnitude; see Fig. 3.

One possible explanation relates to the anisotropy of hand inertia (Hogan 1985; Gordon et al. 1994a; Tsuji et al. 1995). Because of such anisotropy, an external force accelerates the hand in a direction that is not the same as that of the force itself. As a consequence, assistive forces end up in a greater directional error.

\section{Subjects adapt to a constant forces}

We found that movement kinematics under the effect of constant forces changes gradually with practice. More specifically, the endpoint error decreases (see Fig. 4). This suggests that the adapted movements are different from those observed in absence of perturbing forces.

Moreover, a closer look at individual subjects suggests that they use different strategies to adapt to the perturbing force (data not shown). To deal with forces, some subjects increase peak speed, others decrease movement duration, others do both.

These results can be interpreted in the sense that subjects gradually learn to predict the external force. This 
leads to an improved performance (e.g. the decrease in endpoint error). In this, the results are consistent with the findings of (Shadmehr and Mussa-Ivaldi 1994).

\section{EEG recordings}

In terms of EEG correlates, the main findings were the following: (1) during force epochs, just before movement beta band activity increases in proportion to force magnitude. A similar effect was observed in after-CUE alpha band activity in the ipsilateral motor areas; (2) after-CUE alpha band activity in contralateral motor areas decreases with practice; (3) after-CUE theta band activity increases with practice during force trials, irrespective of force magnitude; and (4) theta, alpha, and beta band activity during the after-effect phase changes with respect to the baseline phase.

All the above findings were observed in either T1 or T4 intervals. Some of these effects were also present in the other intervals (T2, T3), but with a lower significance level. A possible explanation lies in the fact that $\mathrm{T} 1$ and $\mathrm{T} 4$ were the closest to the events "CUE" and "GO". Hence, the highest event-related power changes occurred in these intervals, while there was still some residual (or anticipatory) modulation in the $\mathrm{T} 2$ and $\mathrm{T} 3$ intervals.

\section{Beta band activity before movement and after-cue alpha activity reflect force magnitude}

In motor tasks involving force exertion, correlations have been reported between brain activity in beta band and muscle activation. In monkeys performing a precision grip task, Baker et al. (1997) reported a coherence between cortical activity and muscle activation, which reached a maximum in the $20-30 \mathrm{~Hz}$ range. In a tonic contraction task, Mima et al. (1999) reported a synchronization between cortical (beta) and muscle activity, but no relation of beta band activity and force magnitude. In an isometric force compensation task (Chakarov et al. 2009), observed a similar cortico-muscular coherence. In this study, coherence, but not EEG activity, was found to increase at higher force levels.

In contrast, we found that during the force epochs, beta activity before movement increased in proportion to force magnitude; see Fig. 6. It should be noted that in our case, force is a (predictable) perturbation, not a target outcome. Hence, this particular trend may be associated with the prediction of the force needed to complete the movement. An increased beta synchronization suggests a reduced movement-related cortical activity. In particular, our finding may reflect a decreased need for muscle activation (reduced effort) in the presence of assistive forces (greater reduction for greater forces).
We additionally observed an inverse correlation between force magnitude and alpha band synchronization in ipsilateral motor areas: alpha band activity was greater with low forces and smaller with high forces. Similar findings were reported by (Mima et al. 1999) during a tonic contraction task, but in contralateral sensorimotor areas. Event-related rhythm changes in ipsilateral activity have been reported before, but are hard to interpret in this particular context. One possible explanation is that the observed increase in ipsilateral alpha activity reflects cocontraction of the muscles in the resting arm (Waldvogel et al. 2000; Perez and Cohen 2008).

\section{After-cue alpha activity in contralateral motor areas decreases with practice}

EEG correlates of force field adaptation are not well documented in the literature. In the present task, the changes in movement kinematics with practice are small and relatively quick, so no major effect was expected in EEG signals. Nevertheless, we observed a decrease in alpha band synchronization with practice in the contralateral motor area: ERS in the early phases of the force epochs, ERD in later trials. This would suggest a gradual increase in cortical activity. As the effect occurs in the after-CUE interval, it may be related to the gradual development of a capability of predicting the effect of the external force. This is consistent with the observations of (Knyazev et al. 2006) that alpha ERD in the preparation period may be due to an augmented alertness.

It has been suggested that alpha ERS may be due to short-term memory demands (Klimesch et al. 1999) and cortical inhibition preceding the subsequent activation processes (Klimesch et al. 2000). In light of these findings, alternatively, the observed alpha ERS in the early phases of the force epochs might reflect initially higher cognitive demands, which decreased with practice.

\section{After-cue theta activity increases during assisted practice}

It is generally accepted that theta band oscillations reflect performance. More specifically, effective performance has been associated with a phasic increase in theta band activity (Klimesch 1999). It has been suggested that theta ERS may be induced by cognitive processing, especially high memory demands (Klimesch et al. 1996; Jensen and Tesche 2002).

Our results indicate that in the force epochs, theta band synchronization during after-CUE increases in the later force trials, irrespective of the order of presentation of force magnitudes. This observation is consistent with those of (Gevins et al. 1997) who reported that, in a working memory task, theta ERS increases with practice. These 
authors suggested that focusing attention may require more effort after an extended test session. Augmented theta ERS was also reported during exploratory learning in a sensorimotor integration task (Caplan et al. 2003).

In our case, changing forces led subjects to a new situation, thus forcing them to keep attention high. During the second force epoch, subjects might have tried to combine the two previously acquired motor plans (no force and first force) to derive an optimal strategy for the second force, thus the higher theta ERS.

No additional theta ERS increase was observed in the after-effect phase. With force cancelation, subjects might have found themselves in a familiar situation, thus making task execution more automatic and less demanding.

\section{Alpha, beta and theta activity change from baseline to after-effect}

To assess the long-term consequences (after-effects) of force perturbations, we compared the EEG activity during the baseline and after-effect phases. During the after-CUE interval, in the theta band, we observed an increased activity in the left (contralateral) hemisphere and, conversely, a decreased activity in the ipsilateral hemisphere. Moreover, in the same interval, we observed a significant increase in beta band activity over all areas. Finally, in the before-MOV interval, we observed a decrease in alpha band activity over all areas.

The increased theta band activity in the contralateral side denotes a reduced cortical activation after assisted practice. These results may reflect an increased cortical efficiency, or exercise-induced facilitation, in the neural circuitry related to the processing of information about the next target.

In contrast, we found that theta activity decreases in the right (ipsilateral) hemisphere. This result is somewhat consistent with the observation of Gualberto Cremades (2002) that skilled subjects exhibit a greater activity in the alpha band in the left hemisphere as compared to the right, and vice versa for novices. Therefore, this shift of activity from ipsilateral to contralateral might be related to learning and/or a greater expertise.

\section{Conclusions}

Our findings indicate that target-directed, constant-magnitude assistive forces tend to increase extent and directional errors. This finding points to the need that in robot-assisted motor skill learning and rehabilitation applications, the schemes of assistance are carefully designed in order to be effective.

In exercise protocols, an EEG-based BCI could be used to control a robot in order to supplement insufficient muscle control (Buch et al. 2008). However, at least in principle, EEG brain signals could also be used to monitor the current state of patients' brain activity during exercise, as biofeedback to enable patients to modify their abnormal activity, or to modulate the amount of assistance provided by a robot. A pre-requisite is that neural signatures of assistive forces, mental fatigue, and recovery and/or learning must be identified. We found neural signatures for the perceived task difficulty, the progress of adaptation and the magnitude of assistive forces (or, conversely, the amount of voluntary effort). In conclusion, this study is a first step toward extending EEG-based brain-computer interfaces (BCI) from assistive to therapeutic applications (Daly and Wolpaw 2008).

Acknowledgments The research leading to these results has received funding from the European Community's Seventh Framework Programme (FP7/2007-2013) under grant agreement no. FP7ICT-231724 (HUMOUR).

\section{References}

Alegre M, Gurtubay IG, Labarga A, Iriarte J, Malanda A, Artieda J (2003) Alpha and beta oscillatory changes during stimulusinduced movement paradigms: effect of stimulus predictability. Neuroreport 14:381-385

Andrykiewicz A, Patino L, Naranjo JR, Witte M, Hepp-Reymond MC, Kristeva R (2007) Corticomuscular synchronization with small and large dynamic force output. BMC Neurosci 8:101

Baker SN, Olivier E, Lemon RN (1997) Coherent oscillations in monkey motor cortex and hand muscle EMG show taskdependent modulation. J Physiol 501(Pt 1):225-241

Bell AJ, Sejnowski TJ (1995) An information-maximization approach to blind separation and blind deconvolution. Neural Comput 7:1129-1159

Buch E, Weber C, Cohen LG, Braun C, Dimyan MA, Ard T, Mellinger J, Caria A, Soekadar S, Fourkas A, Birbaumer N (2008) Think to move: a neuromagnetic brain-computer interface (BCI) system for chronic stroke. Stroke 39:910-917

Caplan JB, Madsen JR, Schulze-Bonhage A, Aschenbrenner-Scheibe R, Newman EL, Kahana MJ (2003) Human theta oscillations related to sensorimotor integration and spatial learning. J Neurosci 23:4726-4736

Casadio M, Sanguineti V, Morasso PG, Arrichiello V (2006) Braccio di Ferro: a new haptic workstation for neuromotor rehabilitation. Technol Health Care 14:123-142

Casadio M, Morasso P, Sanguineti V, Giannoni P (2009) Minimally assistive robot training for proprioception enhancement. Exp Brain Res 194:219-231

Chakarov V, Naranjo JR, Schulte-Monting J, Omlor W, Huethe F, Kristeva R (2009) Beta-range EEG-EMG coherence with isometric compensation for increasing modulated low-level forces. J Neurophysiol 102:1115-1120

Daly JJ, Wolpaw JR (2008) Brain-computer interfaces in neurological rehabilitation. Lancet Neurol 7:1032-1043

Del Percio C, Infarinato F, Iacoboni M, Marzano N, Soricelli A, Aschieri P, Eusebi F, Babiloni C (2010) Movement-related desynchronization of alpha rhythms is lower in athletes than non-athletes: a high-resolution EEG study. Clin Neurophysiol 121:482-491 
Emken JL, Benitez R, Sideris A, Bobrow JE, Reinkensmeyer DJ (2007) Motor adaptation as a greedy optimization of error and effort. J Neurophysiol 97:3997-4006

Gentili RJ, Bradberry TJ, Hatfield BD, Contreras-Vidal JL (2009) Brain biomarkers of motor adaptation using phase synchronization. Conf Proc IEEE Eng Med Biol Soc 2009:5930-5933

Gevins A, Smith ME, McEvoy L, Yu D (1997) High-resolution EEG mapping of cortical activation related to working memory: effects of task difficulty, type of processing, and practice. Cereb Cortex 7:374-385

Gordon J, Ghilardi MF, Cooper SE, Ghez C (1994a) Accuracy of planar reaching movements. II. Systematic extent errors resulting from inertial anisotropy. Exp Brain Res 99:112-130

Gordon J, Ghilardi MF, Ghez C (1994b) Accuracy of planar reaching movements. I. Independence of direction and extent variability. Exp Brain Res 99:97-111

Gualberto Cremades J (2002) The effects of imagery perspective as a function of skill level on alpha activity. Int J Psychophysiol 43:261-271

Hogan N (1985) The mechanics of multi-joint posture and movement control. Biol Cybern 52:315-331

Hyvarinen A (1999) Fast and robust fixed-point algorithms for independent component analysis. IEEE Trans Neural Netw 10:626-634

Ishii R, Shinosaki K, Ukai S, Inouye T, Ishihara T, Yoshimine T, Hirabuki N, Asada H, Kihara T, Robinson SE, Takeda M (1999) Medial prefrontal cortex generates frontal midline theta rhythm. Neuroreport 10:675-679

Jensen O, Tesche CD (2002) Frontal theta activity in humans increases with memory load in a working memory task. Eur J Neurosci 15:1395-1399

Klimesch W (1999) EEG alpha and theta oscillations reflect cognitive and memory performance: a review and analysis. Brain Res Brain Res Rev 29:169-195

Klimesch W, Doppelmayr M, Russegger H, Pachinger T (1996) Theta band power in the human scalp EEG and the encoding of new information. Neuroreport 7:1235-1240

Klimesch W, Doppelmayr M, Schwaiger J, Auinger P, Winkler T (1999) 'Paradoxical' alpha synchronization in a memory task. Brain Res Cogn Brain Res 7:493-501

Klimesch W, Doppelmayr M, Rohm D, Pollhuber D, Stadler W (2000) Simultaneous desynchronization and synchronization of different alpha responses in the human electroencephalograph: a neglected paradox? Neurosci Lett 284:97-100

Knyazev GG, Savostyanov AN, Levin EA (2006) Alpha synchronization and anxiety: implications for inhibition vs. alertness hypotheses. Int J Psychophysiol 59:151-158

Krebs HI, Palazzolo JJ, Dipietro L, Volpe BT, Hogan N (2003) Rehabilitation robotics: performance-based progressive robotassisted therapy. Auton Robots 15:7-20

Makienko I, Tov EY, Inbar GF (2005) On the effects of adaptation to changing loads on movement-related EEG potentials. Biol Cybern 93:171-177

Marchal-Crespo L, Reinkensmeyer DJ (2009) Review of control strategies for robotic movement training after neurologic injury. J Neuroeng Rehabil 6:20
Mima T, Simpkins N, Oluwatimilehin T, Hallett M (1999) Force level modulates human cortical oscillatory activities. Neurosci Lett 275:77-80

Neuper C, Pfurtscheller G (2001) Event-related dynamics of cortical rhythms: frequency-specific features and functional correlates. Int J Psychophysiol 43:41-58

Neuper C, Wortz M, Pfurtscheller G (2006) ERD/ERS patterns reflecting sensorimotor activation and deactivation. Prog Brain Res 159:211-222

Perez MA, Cohen LG (2008) Mechanisms underlying functional changes in the primary motor cortex ipsilateral to an active hand. J Neurosci 28:5631-5640

Pfurtscheller G, Lopes da Silva FH (1999) Event-related EEG/MEG synchronization and desynchronization: basic principles. Clin Neurophysiol 110:1842-1857

Pfurtscheller G, Stancak A Jr, Neuper C (1996) Post-movement beta synchronization. A correlate of an idling motor area? Electroencephalogr Clin Neurophysiol 98:281-293

Pfurtscheller G, Neuper C, Andrew C, Edlinger G (1997) Foot and hand area mu rhythms. Int J Psychophysiol 26:121-135

Pfurtscheller G, Zalaudek K, Neuper C (1998) Event-related beta synchronization after wrist, finger and thumb movement. Electroencephalogr Clin Neurophysiol 109:154-160

Rearick MP, Johnston JA, Slobounov SM (2001) Feedback-dependent modulation of isometric force control: an EEG study in visuomotor integration. Brain Res Cogn Brain Res 12:117-130

Reinkensmeyer DJ, Patton JL (2009) Can robots help the learning of skilled actions? Exerc Sport Sci Rev 37:43-51

Riener R, Lunenburger L, Jezernik S, Anderschitz M, Colombo G, Dietz V (2005) Patient-cooperative strategies for robot-aided treadmill training: first experimental results. IEEE Trans Neural Syst Rehabil Eng 13:380-394

Shadmehr R, Mussa-Ivaldi FA (1994) Adaptive representation of dynamics during learning of a motor task. J Neurosci 14:3208-3224

Shadmehr R, Wise SP (2005) The computational neurobiology of reaching and pointing : a foundation for motor learning. MIT Press, Cambridge

Slobounov S, Johnston J, Chiang H, Ray W (2002) Movement-related EEG potentials are force or end-effector dependent: evidence from a multi-finger experiment. Clin Neurophysiol 113:1125-1135

Thoroughman KA, Shadmehr R (2000) Learning of action through adaptive combination of motor primitives. Nature 407:742-747

Tsuji T, Morasso PG, Goto K, Ito K (1995) Human hand impedance characteristics during maintained posture. Biol Cybern 72:475-485

Vergaro E, Casadio M, Squeri V, Giannoni P, Morasso P, Sanguineti V (2010) Self-adaptive robot training of stroke survivors for continuous tracking movements. J Neuroeng Rehabil 7:13

Waldvogel D, van Gelderen P, Muellbacher W, Ziemann U, Immisch I, Hallett M (2000) The relative metabolic demand of inhibition and excitation. Nature 406:995-998

Witte M, Patino L, Andrykiewicz A, Hepp-Reymond MC, Kristeva R (2007) Modulation of human corticomuscular beta-range coherence with low-level static forces. Eur J Neurosci 26:3564-3570 